Conclusion Despite in a group education, BDHQ could show the points to change in dietary habits individually. Although how to motivate employees who are entirely uninterested warrant further investigation, using a dietary questionnaire in health education can be an effective way in occupational field.

\section{O8C.6 TOBACCO CONTROL AND ORAL CANCER SCREENING AMONG PUBLIC TRANSPORT BUS DRIVERS, CONDUCTORS AND OTHER STAFF IN MUMBAI, INDIA}

${ }^{1}$ Gauravi Ashish Mishra*, 'Sharmila Pimple, ${ }^{2}$ Anil Singal, ${ }^{1}$ Vasundhara Kulkarni, ${ }^{1}$ Heena Shaikh, ${ }^{1}$ Parishi Majmudar. ${ }^{1}$ Tata Memorial Hospital, Mumbai, India; ${ }^{2}$ BEST, Mumbai, India

\subsection{6/OEM-2019-EPI.201}

Introduction Tobacco is the single most preventable cause of deaths globally. India is the second largest consumer and third largest producer of tobacco. A plethora of tobacco products are consumed. India has highest number of oral cancer cases globally and bears one fourth of the global burden.

With the smoke free public places legislation being implemented since 2nd October 2008, smoking is prohibited on public transport bus premises in India. Tobacco addiction is common among the commuters, drivers, conductors and other staff of the public transport department.

The present study was conducted to understand the knowledge, attitude and practices (KAP) regarding harmful effects of tobacco among the public transport bus staff, to educate them regarding the importance of smoke free legislation and health hazards associated with tobacco use, to support them in tobacco cessation, to conduct oral cancer screening and to assist in the further management.

Materials and methods Around 4000 public transport bus employees in Mumbai were enrolled after explaining the programme and obtaining informed consent. They were interviewed and their KAP regarding tobacco was recorded. The employees were then given detailed health education. They were screened for oral cancers and counseled for tobacco cessation. The screened positive employees were referred for diagnostic evaluation and management.

Results 4000 public transport bus employees were enrolled. Amongst them 1691 (42.28\%) were tobacco users. Smokeless form of tobacco use was dominant, 1561 (92.31\%). All 4000 employees participated in oral cancer screening and were enrolled for tobacco cessation counselling. 743 employees were screened positive, 592 complied to referral for diagnostic evaluation and 534 employees were clinically diagnosed with oral pre-cancers

Conclusions Tobacco use and oral pre-cancers are high among the public transport bus employees in Mumbai. Hence, awareness, oral cavity screening and tobacco cessation counseling should be incorporated within their annual health check-up.

\section{Work Capability}

\section{D.2 OCCUPATIONAL COMPLEXITY IN RELATION TO LATE LIFE PHYSICAL FUNCTIONING IN SWEDEN}

Ingemar Kåreholt*, Alexander Darin Mattsson. Institute of Gerontology, School of Health and Welfare, Jönköping University, Jönköping, Sweden

10.1136/OEM-2019-EPI.202
Background Occupational complexity is a measure of intellectual stimulation at work. Higher occupational complexity has consistently been associated with less cognitive decline, decreased risk of dementia, less psychological distress, and lower mortality. We build on this research by investigating the association between occupational complexity and physical functioning in late life.

Methods Two linked Swedish nationally representative surveys were used. Midlife health, education, social class, income, and occupational complexity from current/latest occupation was assessed in 1991. Data from 1991 also include retrospective questions about life-time occupations. From this data we created an aggregated score (based on occupational complexity at ages $25,30,35,40,45$, and 50 in addition to the first occupation). Trajectories of change in complexity scores were measured using random slope and intercept models. Physical functioning was assessed in 2014 by self-reported mobility limitations and limitations in activities of daily living (ADL).

Results The results show an association between latest and aggregated higher occupational complexity and fewer mobility and ADL limitations. Adjusting for midlife health only moderately affected the associations. The associations were reduced to non-significant when adjusted for education, social class, and income. Trajectories of occupational complexity were only marginally, or not at all, associated with physical functioning in late life.

Discussion Occupational complexity was associated with physical functioning; however, the association could be entirely attributed to socioeconomic position. The initially observed associations between occupational complexity and physical functioning in late life appear to be explained by the same pathways as socioeconomic position. The combined associations between occupational complexity and socioeconomic position on one hand and physical functioning on the other hand need to be further analyzed.

\section{D.3 THE EFFECTIVENESS OF VOCATIONAL REHABILITATION ON WORK PARTICIPATION: A PROPENSITY SCORE MATCHED ANALYSIS USING NATIONWIDE REGISTER DATA}

${ }^{1}$ Taina Leinonen*, 'Eira Viikari-Juntura, 'Kirsti Husgafvel-Pursiainen, ${ }^{1}$ Pirjo Juvonen-Posti, ${ }^{2}$ Mikko Laaksonen, ' Svetlana Solovieva. 'Finnish Institute of Occupational Health, Helsinki, Finland; ${ }^{2}$ Finnish Centre for Pensions, Helsinki, Finland

\subsection{6/OEM-2019-EPI.203}

Introduction Research on the effectiveness of vocational rehabilitation has often focused on small and selected groups of rehabilitees, lacked proper controls, or not captured dynamic changes in work participation. Using rich nationwide data on vocational rehabilitees and their matched controls, we examined long-term changes in work participation before and after vocational rehabilitation to assess its effectiveness.

Methods We used representative Finnish register data to examine 2692 recently employed individuals with histories of musculoskeletal- and mental-related work disability who started vocational rehabilitation in 2008-2010 at age 30-55 (intervention group), and 2692 propensity score matched non-rehabilitees who otherwise met the same criteria (control group). Matching was performed based on sociodemographic and work-related factors, work disability diagnosis, and three-year labour market history. We used generalised estimation equations to examine differences in the 